\title{
The Influence of the Menstrual Cycle on Muscle Strength and Power Performance
}

\author{
by \\ Blanca Romero-Moraleda ${ }^{1}$, Juan Del Coso ${ }^{1}$, Jorge Gutiérrez-Hellín ${ }^{1}$, Carlos Ruiz- \\ Moreno $^{1}$, Jozo Grgic², Beatriz Lara ${ }^{1}$
}

This study aimed to investigate the fluctuations of muscle performance in the Smith machine half-squat exercise during three different phases of the menstrual cycle. Thirteen resistance-trained and eumenorrheic women volunteered to participate in the study $(58.6 \pm 7.8 \mathrm{~kg}, 31.1 \pm 5.5$ years). In a pre-experimental test, the half-squat onerepetition maximum (1RM) was measured. Body mass, tympanic temperature and urine concentration of the luteinizing hormone were estimated daily for 30 days to determine the early follicular phase (EFP), the late follicular phase (LFP), and the mid-luteal phase (MLP) of the menstrual cycle. On the second day of each phase, performance of the Smith machine half-squats was assessed using 20, 40, 60 and 80\% of one repetition maximum (1RM). In each load, force, velocity, and power output were measured during the concentric phase of the exercise by means of a rotatory encoder. The data were analyzed using one-way repeated measures ANOVA coupled with magnitude-based inferences. Overall, force, velocity and power output were very similar in all menstrual cycle phases with unclear differences in most of the pairwise comparisons and effect sizes $>0.2$. The results of this investigation suggest that eumenorrheic females have similar muscle strength and power performance in the Smith machine half-squat exercise during the EFP, $L F P$, and MLP phases of the menstrual cycle.

Key words: women, half-squat exercise, resistance training, periodization, velocity.

\section{Introduction}

During the menstrual cycle, women are exposed to continuous variations in serum concentrations of several female sex steroid hormones. Fluctuations in the main four female sex hormones, namely, estrogen, progesterone, follicle stimulating hormone, and luteinizing hormone are essential to regulate the patterns of the ovulatory cycle. Fluctuations of these hormones also induce two main phases in a regular/normal menstrual cycle: the follicular phase and the luteal phase. Both of these phases are separated by a shorter-time ovulation period in the middle of the cycle. The follicular phase is normally divided into two sub-phases: (a) the early follicular phase (EFP), which is characterized by low serum concentration of both estrogen and progesterone; and (b) the late follicular phase (LFP) where maximal estrogen concentration coincides with low progesterone levels and ends with an upsurge of luteinizing hormone that precedes the ovulation. The luteal phase begins once the luteinizing hormone has returned to basal levels and is characterized by high concentrations of both estrogen and progesterone. Peak values of estrogen and progesterone are generally observed within the mid-term of the luteal phase (MLP).

Estrogen is a hormone with a purported anabolic function, while progesterone has been related to catabolic pathways (Davis and Hackney, 2016). Given these differences in hormone functions, it has been speculated that

1 - Camilo José Cela University. Exercise Physiology Laboratory. Madrid, Spain.

2 - Institute for Health and Sport (IHES), Victoria University, Melbourne, Australia. 
skeletal muscle performance might vary with alterations in hormone production during different phases of the menstrual cycle. However, the current body of literature does not provide clear answers on this topic (Bambaeichi et al., 2004; Fridén et al., 2003; Lebrun et al., 1995). Sarwar et al. (1996) reported an 11\% increase in quadriceps and handgrip maximum voluntary isometric force in the ovulation period which coincided with the LFP. Likewise, Bambaeichi et al. (2004) reported that isometric strength performance peaked during the ovulation phase. These results suggest a potential association between the upsurge of the luteinizing hormone and enhanced muscle strength. However, other studies have not supported these findings as no changes in muscle strength across different phases of the menstrual cycle were found (Fridén et al., 2003; Gür, 1997; Lebrun et al., 1995). For example, Gür (1997) and Janse de Jonge (2003) reported no differences in concentric and eccentric muscle torque between the menstrual, follicular, and luteal phases and similar findings were reported by others (Fridén et al., 2003; Lebrun et al., 1995).

The equivocal evidence might be due to the use of different methods to estimate the phases of the menstrual cycle and the use of different muscle strength performance tests. One possible limitation of the current evidence is that most studies measured maximal/peak values of muscle strength. However, in the practical context, the expression of maximal strength rarely occurs, especially if we consider that resistance training (RT) is commonly performed with submaximal loads (e.g., $80 \%$ of one repetition maximum [1RM]). Also, previous studies used laboratory-based isometric and isokinetic tests of strength. However, in recent years, practitioners have started using velocity data for prediction of and monitoring changes in maximal strength. To the best of our knowledge, there are no studies that explored the effects of different phases of the menstrual cycle on muscle strength and power performance as assessed using barbell velocity measures across low, moderate, and high loading zones. Therefore, the aim of this study was to investigate muscle performance variations across three phases of the menstrual cycle (ELP, LFP, and MLP) by measuring velocity, power and force output in the Smith machine half-squat exercise with loads equivalent to $20,40,60$ and $80 \%$ of
1RM.

\section{Methods}

Participants

Thirteen female triathletes volunteered to participate in this study (age $=31.1 \pm 5.5 \mathrm{yr}$; body mass = $58.6 \pm 7.8 \mathrm{~kg}$; body height $=1.66 \pm 0.06 \mathrm{~m}$; body fat percentage $=14.5 \pm 6.5 \%$ ). All participants had a regular menstrual cycle for the four months previous to the experiment $(27 \pm 2$ days, range $=24-31$ days) and were considered as eumenorrheic. Participants were not using any form of hormonal contraception for at least the six months previous to the experiment. Furthermore, all participants were free from any type of menstrual disorders (e.g., dysmenorrhea, amenorrhea, or heavy symptoms associated with pre-menstrual syndrome), had no musculoskeletal injuries in the three months previous to the investigation and were not taking drugs or dietary supplements during the duration of the experiment. Participants were included if they had at least six months of RT experience (16 \pm 8 months of experience in this sample), and were familiar with the half-squat exercise. During the experimental period, participants were encouraged to maintain their usual training routines. Two weeks before the onset of the experiment protocol, informed consent was obtained from each participant. The experimental protocol was approved by the Human Ethics Committee of the Camilo José Cela University, in accordance with the latest update of the Declaration of Helsinki (2013).

\section{Study design}

In this study, we aimed to investigate the differences in muscle performance in the halfsquat exercise during three different phases of the menstrual cycle. The three phases of the menstrual cycle were EFP, LFP, and MLP, because they represent main events occurring during the menstrual cycle (i.e., menses, pre-ovulation, and peak progesterone concentration, respectively; Janse de Jonge, 2003). To explore this topic, participants performed one repetition (REP) of the Smith machine half-squat exercise at maximal velocity with loads amounting to 20, 40, 60 and $80 \%$ of their respective 1RM (Bazuelo-Ruiz et al., 2015). This assessment was performed in each menstrual cycle phase (Figure 1). The order of the first menstrual cycle phase was randomized for 
each participant and counterbalanced (four started in the EFP, four started in the LFP and the remaining five started in the MLP). The day before each trial, participants were instructed only to perform low-intensity training and maintain their usual diet/fluid intake routine.

\section{Experimental procedures}

Two weeks before the onset of the experiment, participants performed two familiarization sessions with the testing protocol in order to minimize any learning effects during the experiment. One week before the experiment, a 1RM test was performed to standardize the loads in the subsequent experimental sessions. The 1 RM test was performed on a Smith Machine (Technogym, Barcelona, Spain) in which 2 vertical guides regulate the barbell movement. Participants were instructed to perform a countermovement to $90^{\circ}$ of knee flexion until touching a bench and ascend back to an upright position without lifting the toes off the ground. The eccentric phase of the squat was performed in a controlled manner and participants were informed that it should be completed in $\sim 2 \mathrm{~s}$. After $1 \mathrm{~s}$ of maintained knee flexion, the concentric phase of the exercise was completed as fast as possible (2/1/X; Wilk et al., 2019).

Participants commenced the 1RM assessment with sets of increasing loads estimated to be $\sim 20 \%$ 1RM (3-REPs), $\sim 40 \% 1$ RM (3-REPs), $\sim 60 \%$ 1RM (3-REPs), $\sim 80 \%$ 1RM (1-REP), and $\sim 90 \%$ 1RM (1-REP; Banyard, Nosaka and Haff, 2017). This was then followed by the first 1RM attempt with a maximum of five 1RM attempts permitted. After a successful 1RM attempt, the barbell load was increased, in consultation with the participant, between 0.5 and $2.5 \mathrm{~kg}$. The last successful lift with a correct technique was classified as the 1RM load. Two minute passive rest intervals were allocated between all warm-up sets and three minutes of recovery between the 1 RM attempts.

Afterwards, participants performed three identical experimental trials starting with the phase randomly assigned. All three trials were performed in a laboratory, in the morning (between 9 and $11 \mathrm{AM}$ ) and under similar environmental conditions $\left(22-23^{\circ} \mathrm{C}\right.$ and $60 \%$ humidity; OH1001, OH Haus, Spain). Participants arrived at the laboratory in a fed state $(\sim 3$ hours after their last meal). In each experimental trial, the participant was nude-weighed after voiding (Tanita BF 350, Tanita Corporation, Tokyo, Japan). After that, they performed a standardized 15-min warm-up protocol (5-min pedaling on a cycle ergometer at $60 \mathrm{~W}$, followed by mobility exercises and bodyweight half-squats; Banyard et al., 2019). After warming up, participants performed two attempts of the half-squat exercise at maximal velocity with loads that represented 20, 40, 60 and $80 \%$ of their $1 \mathrm{RM}$. All attempts were performed on the same Smith machine that was used for the 1RM assessment. Performance data were measured only during the concentric phase of the movement. Two minute passive rest intervals were allocated between the attempts with the same load and three minutes between different loads. The complete range of motion consisted of lowering the body by bending the knees to a $90^{\circ}$ angle until touching a bench. Execution technique and motivation were standardized and monitored by 2 experienced researchers for greater safety of the participants and reliability of the experimental conditions. In each attempt, barbell displacement (measured in $\mathrm{cm}$ ), mean and peak velocity (MV and $\mathrm{PV}$, respectively; measured in $\mathrm{m} / \mathrm{s}$ ), mean and peak power output (MP and PP; respectively; measured in $\mathrm{W}$ ) as well as mean and peak force (MF and PF, respectively, measured in $\mathrm{N}$ ) were measured in the concentric phase of the movement using a rotatory encoder (Isocontrol, EV-Pro, Spain). The rotatory encoder consists of a velocity sensor connected to the bar by a cable and it records the vertical velocity of the bar during the displacement with a frequency of 500 Hz. This encoder is a reliable system for measuring bar velocity during half-squat exercise. Software associated with the encoder calculated MP and PP from the product of force and bar velocity. This device has been reliably used in previous investigations to assess the effects of caffeine intake on muscle force, velocity and power output (Del Coso et al., 2012). The attempt with the highest barbell displacement velocity in each load was used for further statistical analysis. The 1RM was estimated in all phases to ensure that 1RM remained unchanged during the experiment (Bazuelo-Ruiz et al., 2015).

\section{Determination of the menstrual phase}

The duration of the menstrual cycle and the onset of each phase were determined using: (a) period tracker application; (b) measurement of 
tympanic temperature and body mass changes; and (c) assessment of urinary peak of the luteinizing hormone, following established recommendations (Bambaeichi et al., 2004; Tenan et al., 2013; Tenan et al., 2016). The duration of the menstrual cycle was recorded for a minimum of 4 months for a valid characterization. This information was obtained using a mobile application (Mycalendar ${ }^{\circledR}$, Period-tracker, US) together with a menstruation diary, which included the date of menses, length of menses, and discomfort in the days preceding menses and during the menses. During the familiarization period, participants were informed on how to measure their own basal tympanic temperature and body mass. A digital thermometer (model HDT8208C, Nursal Ear Thermometer, China) and a digital scale (BT200, Daga, Spain) were used for this aim and tympanic temperature and body mass were measured and recorded every morning immediately after waking up (tympanic temperature; EFP: $36.34 \pm 0.42^{\circ}$; LFP: $36.43 \pm 0.62^{\circ}$; MLP: $36.42 \pm 0.47^{\circ}$, body mass; EFP: $58.86 \pm 9.28$ kg; LFP: $58.89 \pm 9.14 \mathrm{~kg}$; MLP: $59.03 \pm 9.11 \mathrm{~kg}$ ). Participants collected these data for one complete menstrual cycle, starting with the phase randomly allocated. In addition, participants were supplied with 7 reactive test strips (One Step Ovulation LH Test Strip; CVS Corporation, US) to assess increases in luteinizing hormone in the firstmorning urine sample. With this information, the beginning of the EFP was indicated by the onset of menses, the LFP was indicated by a positive test for urinary luteinizing hormone (Janse de Jonge, 2003) and the MLP was determined between the 70 and $75 \%$ of the individual menstrual cycle length (i.e., from the $20^{\text {th }}$ to the $22^{\text {nd }}$ day of the menstrual cycle for a regular cycle of 28 days) (Janse de Jonge, 2003). All these protocols helped align the participants' cycle and therefore, despite different cycle lengths, participants performed testing in the same cycle phases.

\section{Statistical analysis}

Data analysis was conducted using the statistical software package SPSS v.20 (SPSS Inc., Chicago, IL, USA). Descriptive statistics included means and standard deviations for all variables. A Shapiro-Wilk test was used to test the data for normality of distribution $(p>0.05)$. The differences in the performance data between the three phases of the menstrual cycle were analyzed using one-way repeated-measures analysis of variance (ANOVA). The statistical threshold was set at $p<0.05$. Additionally, effect sizes (ES) were calculated in all pairwise comparisons to allow a magnitude-based inference approach. Specifically, the effect-size statistic $\pm 90 \%$ confidence interval (CI) was used on log-transformed data to reduce bias due to non-uniformity of error. The smallest significant standardized effect threshold was set as 0.2 , and a qualitative descriptor was included to represent the likelihood of exceeding this threshold. Ranges of likelihood were: $<1 \%$ indicated almost certainly no chances of change; 1 to $5 \%$ indicated very unlikely; 5 to $25 \%$ indicated unlikely; 25 to $75 \%$ indicated possible; 75 to $95 \%$ indicated likely; 95 to $99 \%$ indicated very likely; $>99 \%$ indicated most likely. Differences were rated as unclear when likelihood exceeded $>5 \%$ in both positive/negative directions. ESs were interpreted according to the following ranges: $<0.2$, trivial; $0.2-0.6$, small; $0.6-1.2$, moderate; $1.2-$ 2.0 , large; $2.0-4.0$, very large; and $>4.0$, extremely large (Hopkins et al., 2009).

\section{Results}

The estimated values of 1RM in the halfsquat exercise remained unchanged during the three phases of the menstrual cycle: EFP: $97.0 \pm$ $23.2 \mathrm{~kg}$; LFP: $98.5 \pm 18.1 \mathrm{~kg}$; MLP: $98.1 \pm 22.2 \mathrm{~kg}(p$ $>$ 0.05). One-way ANOVA did not reveal statistically significant differences between the menstrual cycle phases in mean/peak force $(p>$ 0.05 ; Figure 2). At $20 \% 1 \mathrm{RM}, \mathrm{MF}$ was possibly lower in the MLP $(213.8 \pm 68.7 \mathrm{~N})$ than in the LFP $(217.5 \pm 57.1 \mathrm{~N}$; ES: $0.2 \pm 0.3$; chance $\%$ as positive/trivial $/$ negative $=2 / 45 / 53 \%$ ). At $60 \% 1 \mathrm{RM}$, MF was possibly greater during the EFP (539.1 \pm $107.3 \mathrm{~N})$ compared to the MLP $(515.4 \pm 115.5 \mathrm{~N}$; ES: $0.3 \pm 0.5 ; 5 / 35 / 60 \%)$. At $80 \% 1 \mathrm{RM}, \mathrm{MF}$ was possibly lower in the MLP $(656.2 \pm 137.8 \mathrm{~N})$ than the EFP $(677.0 \pm 140.7 \mathrm{~N}$; ES: $0.2 \pm 0.3 ; 2 / 59 / 38 \%)$ and the LFP $(680.8 \pm 124.8 \mathrm{~N}$; ES: $0.2 \pm 0.3$; $3 / 56 / 41 \%$ ). At $60 \% 1 \mathrm{RM}$, PF was possibly greater in the EFP $(614.6 \pm 121.6 \mathrm{~N})$ in comparison with the MLP $(599.9 \pm 150.8 \mathrm{~N}$; ES: $0.2 \pm 0.2 ; 3 / 31 / 66)$. At $80 \% 1 \mathrm{RM}$, PF was possibly greater in the LFP $(803.7 \pm 168.7 \mathrm{~N})$ compared to the EFP $(792.2 \pm$ $170.4 \mathrm{~N}$; ES: $0.2 \pm 0.2 ; 42 / 57 / 0)$ and the MLP (776.6 $\pm 172.6 \mathrm{~N}$; ES: $0.2 \pm 0.4 ; 5 / 47 / 48$ ).

One-way ANOVA did not reveal 
statistically significant differences between the three phases of the menstrual cycle in mean/peak velocity (Figure 3). At 20\% 1RM, MV was possibly lower in the MLP $(0.7 \pm 0.1 \mathrm{~m} / \mathrm{s})$ than the EFP $(0.72$ $\pm 0.10 \mathrm{~m} / \mathrm{s}$; ES: $0.2 \pm 0.4 ; 4 / 44 / 51 \%)$ and the LFP $(0.7$ $\pm 0.1 \mathrm{~m} / \mathrm{s}$; ES: $0.4 \pm 0.4 ; 2 / 23 / 75 \%$ ). In addition, at $60 \% 1 \mathrm{RM}, \mathrm{MV}$ was possibly greater during the EFP $(0.56 \pm 0.07 \mathrm{~m} / \mathrm{s})$ compared to the MLP $(0.5 \pm$ $0.1 \mathrm{~m} / \mathrm{s}$; ES: $0.3 \pm 0.4 ; 2 / 34 / 64 \%)$ and the LFP $(0.5 \pm$ $0.1 \mathrm{~m} / \mathrm{s}$; ES: $0.2 \pm 0.4 ; 3 / 42 / 55 \%)$. At $40 \% 1 \mathrm{RM}, \mathrm{PV}$ was possibly greater in the MLP $(1.3 \pm 0.2 \mathrm{~m} / \mathrm{s})$ compared to the LFP $(1.2 \pm 0.2 \mathrm{~m} / \mathrm{s}$; ES: $0.3 \pm 0.3$; 65/34/1).

One-way ANOVA did not reveal statistically significant differences between the three phases of the menstrual cycle phases in mean/peak muscle power (Figure 4). At 20\% 1RM, MP was possibly greater in the MLP $(162.8 \pm 60.6 \mathrm{~W})$ compared to the LFP (155.8 $\pm 49.2 \mathrm{~W}$; ES: $0.2 \pm 0.4 ; 3 / 40 / 57)$; at $40 \% 1 \mathrm{RM}$, MP was most likely greater also in the MLP $(242.6 \pm 68.6 \mathrm{~W})$ compared to the EFP (159.9 \pm 66.7 W; ES: $0.8 \pm 0.3 ; 100 / 0 / 0)$; at $60 \% 1 \mathrm{RM}$, MP was possibly greater in the EFP $(299.0 \pm 70.34 \mathrm{~N})$ compared to the MLP $(283.8 \pm 84.4 \mathrm{~W}$; ES: $0.3 \pm$ $0.4 ; 4 / 28 / 68)$. PP was most possibly greater in the MLP $(462.0 \pm 151.8 \mathrm{~W})$ than the LFP $(450.6 \pm 150.3$ W; ES: $0.2 \pm 0.2 ; 36 / 63 / 1)$ at $40 \% 1 \mathrm{RM}$.

\section{Discussion}

The aim of this study was to investigate muscle force $(\mathrm{N})$, velocity $(\mathrm{m} / \mathrm{s})$ and power output (W) variations across three different phases of the menstrual cycle in resistance exercise performed with loads equivalent to 20, 4060 and $80 \%$ of $1 \mathrm{RM}$. Overall, the results of this investigation indicate that there were no systematic variations in muscle performance during the menstrual cycle, as measured in a wide range of loads (from 20 to $80 \%$ of $1 \mathrm{RM}$ ). The lack of an effect of the menstrual cycle on muscle performance was evident for mean and peak values of force, velocity, and power output which therefore suggests that muscle strength and power performance of eumenorrheic women are not affected by different phases of the menstrual cycle.

Our findings are consistent with outcomes of previous studies (Fridén et al., 2003; Janse de Jonge et al., 2001; Lebrun et al., 1995; Montgomery and Shultz, 2010) in which researchers reported no differences in maximal force among menstrual cycle phases. However, muscle strength tests most commonly used in previous studies included the handgrip test and isokinetic testing for knee flexors and extensors. Both tests are valid for the measurement of maximal force values, but they do not provide information related to the association of force and velocity in submaximal loading. We selected the half-squat exercise with increasing loads to determine whether the menstrual cycle affected muscle performance in a wide range of low-tohigh loads. The variables measured in this study to assess muscle performance (i.e., velocity of the barbell displacement as well as force and power output generated during the concentric phase of the Smith machine half-squat) have a greater practical implication for monitoring training loads than traditional percentage-based loading methods (Dorrel et al., 2019). To the best of our knowledge, our study is the first that explored the effects of the menstrual cycle on force and power output using loads of increasing intensity (from 20 to $80 \%$ of $1 \mathrm{RM}$ ).

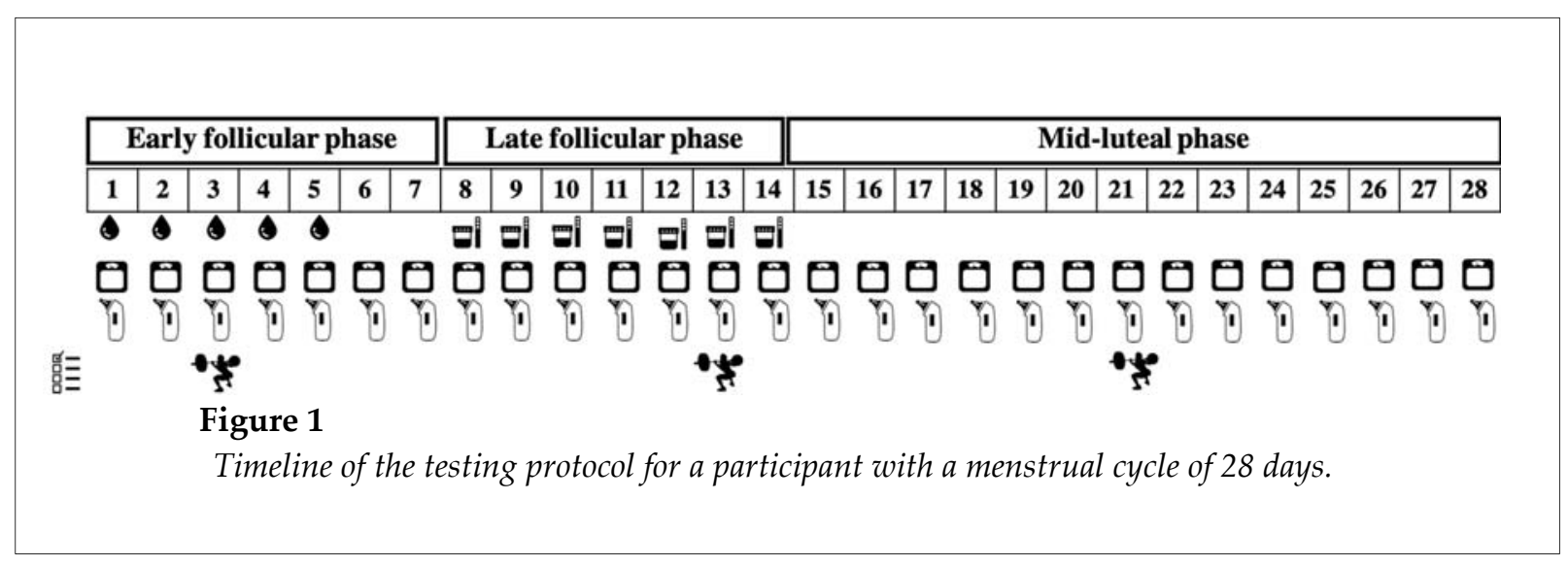

(C) Editorial Committee of Journal of Human Kinetics 

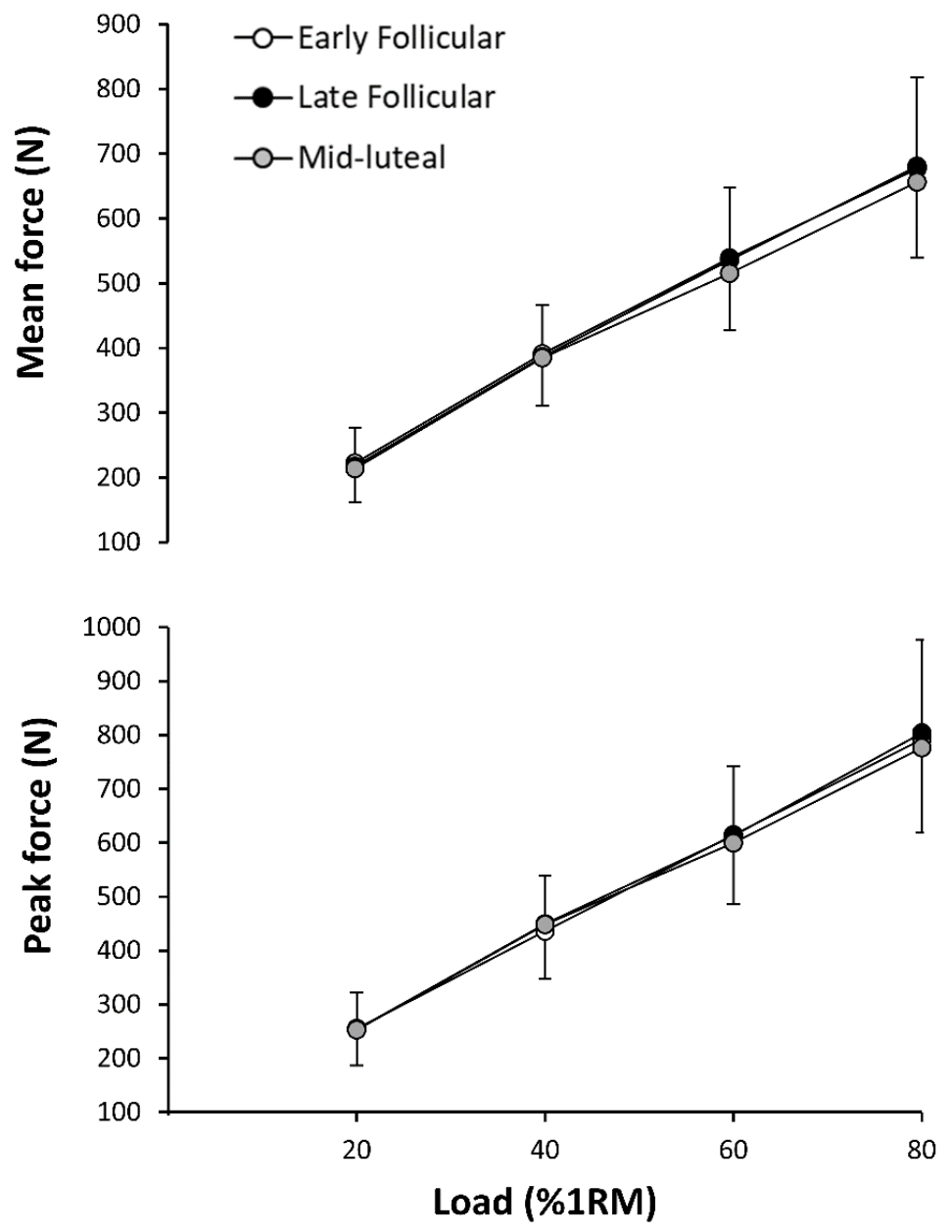

Figure 2

Mean (upper panel) and peak force (lower panel) during the concentric phase of Smith machine half-squat exercise at increasing intensity (20, 40, 60 and 80\% of 1RM) in each phase of the menstrual cycle. Each symbol is the mean \pm standard deviation. 

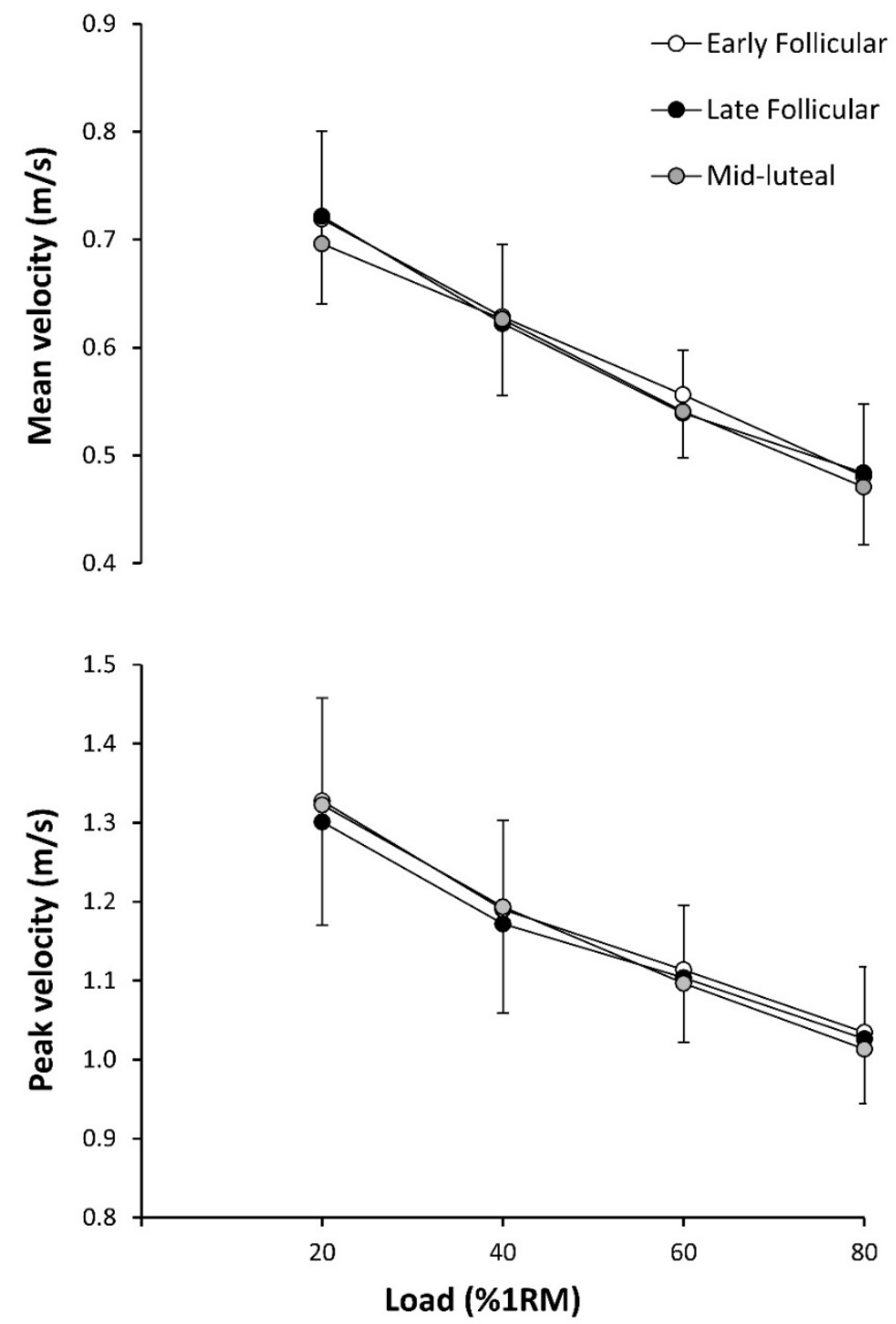

Figure 3

Mean (upper panel) and peak velocity (lower panel) of the barbell during the concentric phase of Smith machine half-squat exercise at increasing intensity (20, 40, 60 and 80\% of 1RM) in each phase of the menstrual cycle. Each symbol is the mean \pm standard deviation. 

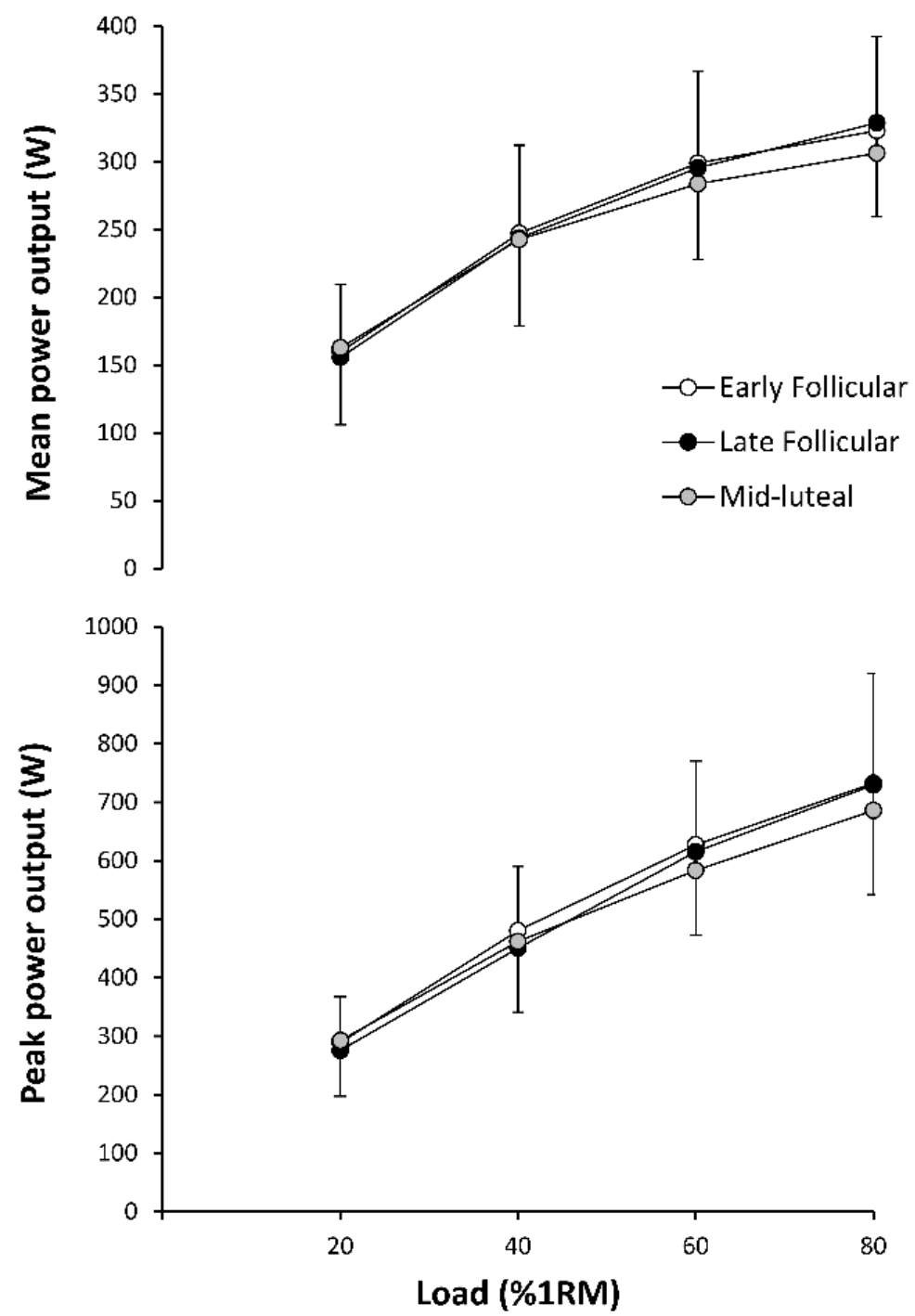

Figure 4

Mean (upper panel) and peak power output (lower panel) during the concentric phase of Smith machine half-squat exercise at increasing intensity (20, 40, 60 and 80 of 1RM) in each phase of the menstrual cycle. Each symbol is the mean \pm standard deviation.

Given the lack of studies using similar performance tests, the comparison of the results presented herein with other investigations is difficult. Nonetheless, our results support those of previous studies (that used isometric/isokinetic tests) which indicate that different phases of the menstrual cycle do not affect muscle strength.

Studies using transcranial magnetic stimulation (Smith et al., 1989, 1999) indicate that the LFP (when estradiol reaches peak concentration) is coupled with enhanced cortical excitation, in contrast to the EFP and the luteal phase that are characterized by cortical inhibition (Smith et al., 1989, 1999). This may be important to emphasize given the clear positive association between excitability and muscle strength (Flanagan et al., 2012). Therefore, a logical hypothesis here is that muscle strength should be 
increased during the LFP due to the increase in excitation and reduced in the EFP due to cortical inhibition. However, the results obtained in the present study do not support this notion as force production was comparable in all menstrual cycle phases. The results did not show any statistically significant differences in MF and PF, velocity, nor power output. In addition, the magnitude-based inference analysis showed small ES magnitudes. Based on these findings, it seems that the mean and peak force, velocity and power output performance in the Smith machine half-squat for eumenorrheic women is not affected by different phases of the menstrual cycle. However, these results might not be applicable to athletes with dysmenorrhea and premenstrual syndrome (Czajkowska et al., 2015) and further investigation is warranted on women with irregular/abnormal menstrual cycles.

While we did not find any significant differences in exercise performance in our sample, these results do not dispute the notion that hormone fluctuations during the menstrual cycle may influence hallmark adaptations to long-term RT such as increased strength and muscle hypertrophy. In fact, this speculation has been confirmed by two previous investigations. In these studies, changes in maximum isometric muscle force and muscle diameters (Sung et al., 2014) were greater when the frequency of RT was high in the follicular phase and low in the luteal phase as compared to training with a low frequency in the follicular phase and high in the luteal phase. These results are likely based on the anabolic properties of estrogen, suggesting that a positive anabolic hormone milieu coupled with more often increases in muscle protein synthesis (via increased training frequency) favor gains in muscle mass during the late follicular phase (Sung et al., 2014; Wikström-Frisén et al., 2017).

\section{Acknowledgements}

The authors would like to thank the participants for their invaluable contribution in this study.

\section{References}

Bambaeichi E, Reilly T, Cable N, Giacomoni M. The Isolated and Combined Effects of Menstrual Cycle Phase and Time-of-Day on Muscle Strength of Eumenorrheic Females. Chronobiol Int, 2004; 21: 645-60

Banyard H, Nosaka K, Haff G. Reliability and Validity of the Load-Velocity Relationship to Predict the 1RM Back Squat. J Strength Cond Res, 2017; 31: 1897-1904

Banyard H, Tufano J, Delgado J, Thompson S, Nosaka K. Comparison of the Effects of Velocity-Based Training Methods and Traditional 1RM-Percent-Based Training Prescription on Acute Kinetic and
Considering results of these two investigations, together with the findings of our study, it seems the improved RT adaptations observed in those two studies are the result of the anabolic effect of estrogen (Enns and Tiidus, 2010) rather than an improved muscle capacity for training in the follicular phase.

There are several limitations to this study that should be mentioned and discussed. Firstly, to determine the onset of the menstrual cycle application and measured changes in tympanic temperature and body mass. In addition, we also used luteinizing hormone urine test strips, as previously recommended (Bambaeichi et al., 2004; Tenan et al., 2013, 2016). This is a valid methodology and was used in previous studies (Bambaeichi et al., 2004; Pallavi et al., 2017), however, there was no measurement of serum female sex hormones to confirm the cycle length and the beginning/end of each phase. Secondly, although the individuals who participated in this study had at least six months of RT experience, they had no experience in velocity-based training. Finally, although none of our participants reported heavy menstrual bleeding, we did not obtain data about the exact amount of blood lost during menstruation. Given that this may be important, future investigations on this topic should consider including this measurement as well.

In conclusion, the results of this study suggest that resistance-trained eumenorrheic females have similar velocity, force, and power performance when exercising with loads equivalent to $20,40,60$ and $80 \%$ of $1 \mathrm{RM}$ in the Smith machine half-squat exercise during the different phases of the menstrual cycle. phases we used a menstrual period tracker 
Kinematic Variables. Int J Sports Physiol Perform, 2019; 14: 246-255

Bazuelo-Ruiz B, Padial P, García-Ramos A, Morales-Artacho AJ, Miranda MT, Feriche B. Predicting Maximal Dynamic Strength from the Load-Velocity Relationship in Squat Exercise. J Strength Cond Res, 2015; 29: 1999-2005

Czajkowska M, Drosdzol-Cop A, Gałazka I, Naworska B, Skrzypulec-Plinta V. Menstrual Cycle and the Prevalence of Premenstrual Syndrome/Premenstrual Dysphoric Disorder in Adolescent Athletes. J Pediatr Adolesc Gynecol, 2015; 28: 492-498

Davis HC, Hackney AC. The Hypothalamic-Pituitary-Ovarian Axis and Oral Contraceptives: Regulation and Function. In Sex Hormones, Exercise and Women: Scientific and Clinical Aspects, Springer, 1-17; 2016

Del Coso J, Salinero JJ, González-Millán C, Abián-Vicén J, Pérez-González B. Dose Response Effects of a Caffeine-Containing Energy Drink on Muscle Performance: A Repeated Measures Design. J Int Soc Sports Nutr, 2012; 9: 21

Dorrel HF, Smith M, Gee T. Comparison of Velocity-Based and Traditional Percentage-Based Loading methods on Maximal Strength and Power Adaptations. J Strength Cond Res, 2019; doi: 10.1519/JSC.0000000000003089. [Epub ahead of print]

Enns DL, Tiidus PM. The Influence of Estrogen on Skeletal Muscle: Sex Matters. Sport Med, 2010; 1: 41-58

Flanagan SD, Dunn-Lewis C, Comstock BA, Maresh CM, Volek JS, Denegar CR, and Kraemer WJ. Cortical Activity during a Highly-Trained Resistance Exercise Movement Emphasizing Force, Power or Volume. Brain Sci, 2012; 2: 649-66

Fridén C, Hirschberg A, Saartok T. Muscle Strength and Endurance Do Not Significantly Vary Across 3 Phases of the Menstrual Cycle in Moderately Active Premenopausal Women. Clin J Sport Med, 2003; 13: $238-241$

Gür H. Concentric and Eccentric Isokinetic Measurements in Knee Muscles during the Menstrual Cycle: A Special Reference to Reciprocal Moment Ratios. Arch Phys Med Rehabil, 1997; 78: 501-505

Hopkins WG, Marshall S, Batterham A, Hanin J. Progressive Statistics for Studies in Sports Medicine and Exercise Science. Med Sci Sports Exerc, 2009; 41: 3-12

Janse de Jonge, X. Effects of the Menstrual Cycle on Exercise Performance. Sport Med, 2003; 33: 833-851

Janse de Jonge X, Boot C, Thom J, Ruell P, Thompson M. The Influence of Menstrual Cycle Phase on Skeletal Muscle Contractile Characteristics in Humans. J Physiol, 2001; 530: 161-166

Lebrun C, McKenzie D, Prior J, Taunton J. Effects of Menstrual Cycle Phase on Athletic Performance. Med Sci Sports Exerc, 1995; 27: 437-444

Montgomery M, Shultz SJ. Isometric Knee-Extension and Knee-Flexion Torque Production During Early Follicular and Postovulatory Phases in Recreationally Active Women. J Athl Train, 2010; 45: 586-593

Pallavi LC, Souza J, Shivaprakash G. Assessment of Musculoskeletal Strength and Levels of Fatigue During Different Phases of Menstrual Cycle in Young Adults. J Clin Diagnostic Res, 2017; 11: CC11-13

Sarwar R, Niclos B, Rutherford O. Changes in Muscle Strength, Relaxation Rate and Fatiguability During the Human Menstrual Cycle. J Physiol, 1996; 493: 267-272

Smith MJ, Keel J, Greenberg B, Adams L., Schmidt PJ, Rubinow D, Wassermann E. Menstrual Cycle Effects on Cortical Excitability. Neurology, 1999; 53: 2069-2072

Smith SS, Woodward DK, Chapin J. Sex Steroids Modulate Motor-Correlated Increases in Cerebellar Discharge. Brain Res, 1989; 476: 307-316

Sung E, Han A, Hinrichs T, Vorgerd M, Manchado C, Platen P. Effects of Follicular Versus Luteal PhaseBased Strength Training in Young Women. Springerplus, 2014; 3: 668

Tenan M, Hackney AC, Griffin L. Maximal Force and Tremor Changes Across the Menstrual Cycle. Eur J Appl Physiol, 2016; 116: 153-60 
Tenan M, Peng YL, Hackney AC, Griffin L. Menstrual Cycle Mediates Vastus Medialis and Vastus Medialis Oblique Muscle Activity. Med Sci Sports Exerc, 2013; 45: 2151-2157

Wikström-Frisén L, Boraxbekk C, Henriksson-Larsén K. Effects on Power, Strength and Lean Body Mass of Menstrual/Oral Contraceptive Cycle Based Resistance Training. J Sports Med Phys Fitness, 2017; 57: 4352

Wilk M, Golas A, Krzysztofik M, Nawrocka M, Zajac A. The Effects of Eccentric Cadence on Power and Velocity of the Bar during the Concentric Phase of the Bench Press Movement. J Sport Sci Med, 2019; 18: 191-197

\section{Corresponding author:}

Juan Del Coso. https://orcid.org/0000-0002-5785-984X

Camilo José Cela University.

C/ Castillo de Alarcon, 49. Villafranca del Castillo, 28692. SPAIN

Phone: 34+918 153131 (Ext. 1627)

Fax.: 34+918 153131

E-mail: jdelcoso@ucjc.edu 